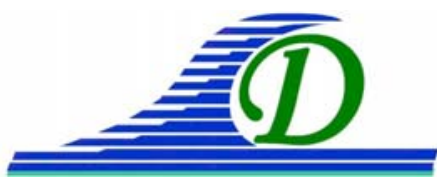
XIII ${ }^{\text {èmes }}$ Journées Nationales Génie Côtier - Génie Civil Dunkerque, 2-4 juillet 2014

DOI: 10.5150/jngcgc.2014.035 (c) Editions Paralia CFL disponible en ligne - http://www.paralia.fr - available online

\title{
Evolution diachronique de la ligne du rivage le long des littoraux méditerranéens occidentaux marocains : cas des sites côtiers de Ksar Esghir, Bou Ahmed et Jebha (Maroc)
}

\author{
Brahim EL MOUTCHOU $^{1}$, Hanaa BENALI ${ }^{1}$, Amal MAMOUNI ${ }^{1}$
}

1. Equipe de Recherche en Géologie et Océanologie (ERGO/UAE/E02FS), Département de Géologie, Faculté des Sciences. BP 2121, M’hannech. 2. 93000 Tétouan, Maroc. brahimelmoutchou@yahoo.fr ; hanabena@gmail.com ; mamouni.amal@gmail.com

\section{Résumé :}

L'étude de l'évolution diachronique par comparaison entre des traits de côte de sites côtiers, au Nord-Ouest du Maroc, dans la région de Ksar Esghir sur le détroit du Gibraltar entre Tanger et Sebta et dans la région de Bou Ahmed et Jebha le long des côtes méditerranéennes a permis d'apprécier, de qualifier et de quantifier, l'évolution historique de la ligne de rivage entre 1966 et 2005 pour la région de Ksar Esghir et entre 1969 et 2009 pour les régions de Bou Ahmed et Jebha, avec :

- une érosion des plages de : Ksar Esghir, avec un recul du trait de côte de -28 m sur 40 ans, soit un taux moyen annuel de $-0.7 \mathrm{~m} / \mathrm{an}$, Stehat (Bou Ahmed), d'environ 29,8 $\mathrm{m}$ sur 41 ans, soit un taux moyen annuel de l'ordre de -0,7 m/an et Jebha avec un recul de -30,0 m sur 41 ans, soit un taux moyen annuel de l'ordre de -0,7 m/an,

- une accrétion des plages de: Ksar Esghir, avec une avancée du trait de côte de $+23.1 \mathrm{~m}$ sur 40 ans, soit un taux moyen annuel de $+0,6 \mathrm{~m} / \mathrm{an}$, au nord de l'embouchure de l'oued Tighissasse (Chmaala à Bou Ahmed), avec un taux global de $+86,5 \mathrm{~m}$ sur 41 ans, soit un taux moyen annuel de l'ordre de $+2,1 \mathrm{~m} / \mathrm{an}$ et au nord de l'embouchure de l'oued Ouringa (Jebha) avec un taux global de $+62,4 \mathrm{~m}$ sur 41ans, soit un taux moyen annuel de l'ordre de $+1,5 \mathrm{~m} / \mathrm{an}$,

- une stabilité limitée.

Mots-clés : Evolution littorale, Erosion, Accrétion, Tanger, Ksar Esghir, Maroc.

\section{Introduction}

Les zones côtières, sous la dépendance des pressions démographique, économique et écologique, connaissent des variations morphodynamiques dont les plus marquées sont les phénomènes d'érosion. La gestion des risques d'érosion des zones côtières s'imposent aujourd'hui comme un axe stratégique dans les concepts d'aménagement du territoire et de développement durable. Ainsi, la ligne du rivage est en perpétuelles modifications liées essentiellement aux effets des agents dynamiques marins,continentaux et/ou anthropiques. L'étude de son évolution permet de comprendre les impacts exercés par ces agents à ce niveau. 


\section{Thème 2 - Dynamique sédimentaire}

Dans ce contexte, les régions de Ksar Esghir, Bou Ahmed et Jebha par leurs positions stratégiques sont le siège d'un certain nombre d'activités socio-économiques (activités touristiques, industrielles et logistiques portuaires, ...). L'étude de l'évolution historique de ces sites le long du littoral méditerranéen marocain, a permis d'appréhender les tendances et les impacts qu'exercent les divers agents sur ce milieu.

\section{Caractères généraux de la zone d'étude}

Situés au Nord-Ouest du Maroc, les sites de Ksar Esghir, Bou Ahmed et Jebha, font partie de la chaine du Rif, qui est l'un des segments de la chaîne alpine constituant le tronçon Sud de l'arc de Gibraltar (DURAND DELGA et al., 1960-1962 ; DIDON et al., 1973 ; DURAND DELGA \& FONTBOTE, 1980). Le Rif est formé de l'empilement des nappes des Sebtides et de quelques affleurements des Ghomarides et de la Dorsale calcaire. Ces unités sont affectées par une succession de phases tectoniques à l'origine des plis de direction SW-NE et SE-NW (CHALOUAN, 1986; BENMAKHLOUF, 1990).

Ces sites côtiers, d'une direction générale E - W à NW - SE, sont limités au Nord par le Détroit de Gibraltar, à l'Ouest et au Sud par les formations géologiques de la chaîne du Rif et à l’Est par la Mer Méditerranée.

Cette région présente trois entités morphologiques distinctes à savoir : les reliefs élevés, les plaines basses fluvio-marines et les côtes sableuses (figure 1).

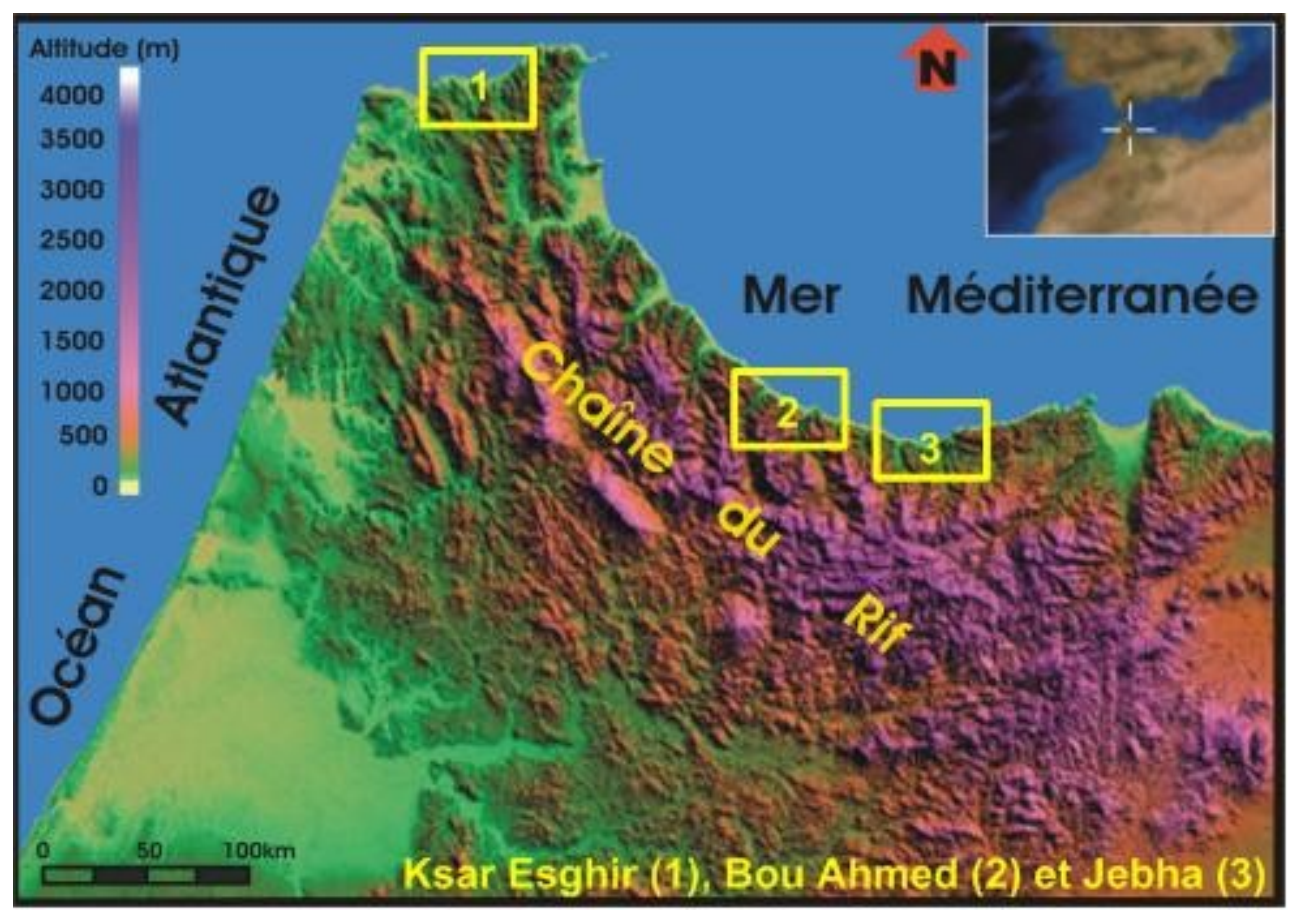

Figure 1. Aspects géomorphologiques des régions étudiées. 


\section{XIII ${ }^{\text {èmes }}$ Journées Nationales Génie Côtier - Génie Civil \\ Dunkerque, 2-4 juillet 2014}

Le contexte climatique est de type méditerranéen caractérisé par un climat tempéré et chaud à deux saisons distinctes: Un hiver frais et humide aux précipitations le plus souvent brutales, qui s'étend d'octobre à avril et un été chaud et sec de mai à septembre. Ce contexte climatique est marqué par une pluviométrie moyenne annuelle comprise entre $470 \mathrm{~mm}$ (Stehat - Jebha) et 800 (Ksar Esghir), une température moyenne annuelle d'environ $18{ }^{\circ} \mathrm{C}$ et des vents à deux composantes Est et Ouest (figure 2).

Le contexte hydrodynamique dans le Détroit de Gibraltar (région de Ksar) et la Méditerranée (région de Bou Ahmed et Jebha) est marqué par des houles qui proviennent essentiellement de deux secteurs (JAAIDI et al., 1993 ; EL MOUTCHOU, 1995 ; EL FADEL, 2006 ; EL MEKADEM et al., 2009 ; NHALA, 2013) (figure 3), avec :

- des houles Ouest à NW dominantes d'origine atlantique sur la partie occidentale, où les périodes varient entre 9 et $12 \mathrm{~s}$, avec une amplitude moyenne de $2 \mathrm{~m}$. Suite à la réfraction, ces houles, atteignent les côtes en générant un courant latéral vers l'ouest,

- un hydrodynamisme dominé par les effets conjugués des houles et des marées sur ces côtes méditerranéennes de la partie méditerranéenne. L’onde de marée, généralement très faible, provient de l'Atlantique et se propage vers la Méditerranée à travers le détroit de Gibraltar. Elle est de type semi-diurne à faible marnage qui décroît de l'Ouest vers l'Est (EL MOUTCHOU, 1995 ; EL MOUTCHOU et al., 2011a \& 2011b).

- L’hydrographie de la région est caractérisée par un réseau hydrographique dense, serré et à régime d'écoulement irrégulier permanent matérialisé par deux principaux cours d'eau dans la région de Bou Ahmed, avec : Oued Tighissasse et ses deux afluents El Kanar et Bouhya, à bassin versant d'une superficie de $633 \mathrm{~km}^{2}$ et Oued Ouringa dans la région de Jebha à bassin versant d'une superficie de $510 \mathrm{~km}^{2}$ (EL MAKADEM et al., 2009). Pour la région de Ksar Esghir, les principaux cours d'eau sont : Oueds Rmel, Ksar Esghir et Lmarse.

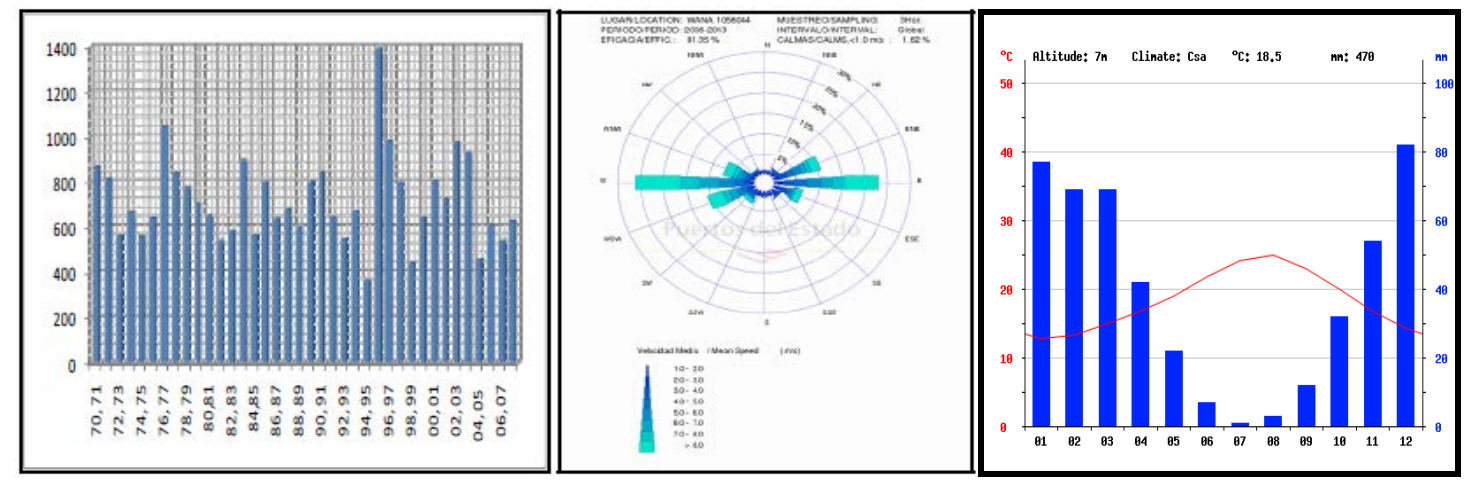

Figure 2. Distribution des précipitations annuelles et rose des vents à Ksar Esghir

(Station kalaya) et courbe ombrothermique de Stehat/Jebha

(www.puerto.es et www.climate-data.com). 


\section{Thème 2 - Dynamique sédimentaire}

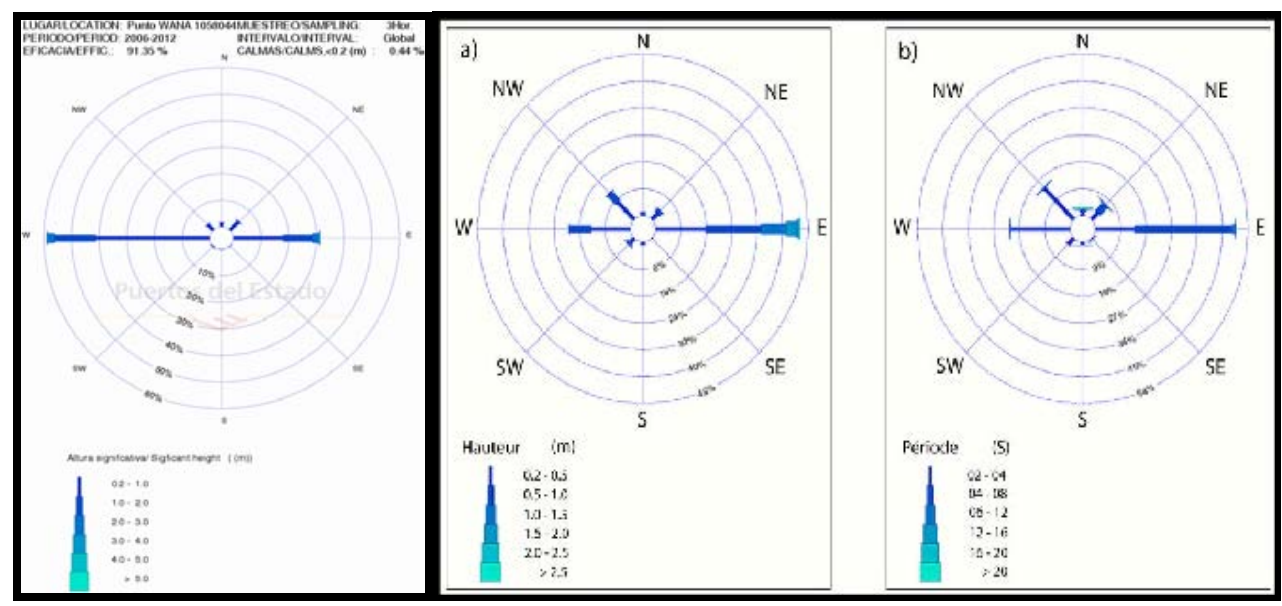

Figure 3. Rose des houles à Ksar Esghir / Sebta (point WANA 1058044) et sur les côtes méditerranéennes entre Tétouan / et Bou Ahmed (point WANA 2006006)

(Source : http//:www.puerto.es).

\section{Méthodes et techniques d'étude}

L'ensemble des documents cartographiques utilisés pour cette analyse se présentent comme suit (tableau 1). Le traitement de ces documents a été effectué en plusieurs étapes à savoir : le mosaïquage et le géoréférencement des cartes utilisées, la digitalisation, la qualification et la quantification des tendances évolutives des lignes du rivage des trois secteurs considérés le long de cette frange littorale.

Suite à ce traitement, on procède à la superposition des différentes couches vectorielles obtenues puis au calcul des distances séparant les traits de côtes de chaque couche. Ainsi, ces taux moyens du recul et/ou d'engraissement du trait de côte sont calculés à partir de la mesure des écarts entre les traits de côtes, en se basant sur la formule suivante (EL MEKADEM et al., 2009 ; EL MOUTCHOU et al., 2011a et 2011b), avec :

$M=\Sigma \Delta d / N$

$M$ : la moyenne générale de recul et/ou engraissement (en mètre linéaire)

$\Sigma \Delta d$ : la somme des écarts entre les distances mesurées entre les traits de côte

$N$ : le nombre total des mesures effectuées par site

Tableau 1. Documents utilisés dans l'étude de l'évolution historique.

\begin{tabular}{|c|c|c|c|}
\hline Documents & Feuilles topographiques & Année & Sources \\
\hline \multirow{6}{*}{$\begin{array}{l}\text { Cartes } \\
\text { topographiques }\end{array}$} & $\begin{array}{l}\text { Ksar Esghir et Sebta au } \\
1 / 50000^{\text {ème }}\end{array}$ & $1966-1970$ & \multirow{6}{*}{ Division de la Carte Rabat - Maroc } \\
\hline & Tétouan et Tanger $1 / 100000^{\text {ème }}$ & $1972-1974$ & \\
\hline & $\begin{array}{l}\text { Hjar Esfar, Ksar Esghir et Port } \\
\text { Tanger Med au 1/25000ème }\end{array}$ & 2005 & \\
\hline & Bou Ahmed - Jebha 1/50000 & 1969 & \\
\hline & Bou Ahmed - Jebha au 1/25000 ème & 2009 & \\
\hline & Nord du Maroc $1 / 50000^{\text {ème }}$ & 1985 & \\
\hline
\end{tabular}




\section{XIII ${ }^{\text {èmes }}$ Journées Nationales Génie Côtier - Génie Civil \\ Dunkerque, 2-4 juillet 2014}

\section{Résultats - Discussions}

La superposition des traits de côte obtenus, à partir des documents cartographiques montre trois contextes évolutifs différents (érosion, engraissement et stabilité du trait de côte). La synthèse de ces évolutions pour les différents sites est présentée dans la figure 4 et le tableau 2 en allant de l'Ouest vers l'Est.

\subsection{Bilan évolutif des littoraux de la région de Ksar Esghir entre 1966 et 2005}

La synthèse de ces évolutions pour les différents sites, de la région de Ksar Esghir, sont présentés dans la figure 4 et le tableau 2, avec :

- une stabilité très limitée entre Ras Cires et Ksar Esghir (Zone 2), zone englobant le site du port Tanger - Med,

- une accrétion avec un taux moyen annuel de l'ordre de $+0,6 \mathrm{~m} / \mathrm{an}$, touchant les plages : à l'Est de Ksar Esghir (Zone 2), entre Maki el Maaz et Lamrissa Sghira (Zone 3), entre oued Allyenne et Lahjer Lasfar (Zone 3), et à Sidi Qanqouch et Playa Blanca (Zone 4),

- une érosion quasi-totale, avec un taux moyen annuel de l'ordre de -0,7 m/an, touchant les plages : Entre Ras Leone et Ras Cires (Zone 1), l’Ouest de Ksar Esghir (Zone 2), les sites Eddiki et Maki Elmaaz, entre Lmarssa Sghira et Lhajar Lasfar (Zone 3) et à Ain Boumaaza, Playa Blanca et Lamnar (Zone 4).

Tableau 2. Evolution du trait de côte des régions de Ksar Esghir entre 1966 et 2005, Bou Ahmed (Stehat et Chmaala) et Jebha entre 1969 et 2009.

\begin{tabular}{|c|c|c|c|c|}
\hline & Zones & $\begin{array}{l}\text { Taux global moyen } \\
\text { (m) }\end{array}$ & $\begin{array}{l}\text { Taux moyen annuel } \\
\text { (m/an) }\end{array}$ & Constats \\
\hline \multirow{8}{*}{$\begin{array}{l}\text { région de Ksar Esghir } \\
\text { (Sur } 40 \text { ans) }\end{array}$} & Zone 1 & $-23,5$ & $-0,6$ & Erosion \\
\hline & \multirow{3}{*}{ Zone 2} & $-28,2$ & $-0,7$ & Erosion \\
\hline & & $+16,7$ & $+0,4$ & Accrétion \\
\hline & & 0,0 & 0,0 & Stabilité \\
\hline & \multirow{2}{*}{ Zone 3} & $-33,3$ & $-0,8$ & Erosion \\
\hline & & $+24,5$ & $+0,6$ & Accrétion \\
\hline & \multirow{2}{*}{ Zone 4} & $-27,0$ & $-0,7$ & Erosion \\
\hline & & $+28,4$ & $+0,7$ & Accrétion \\
\hline \multirow{3}{*}{$\begin{array}{l}\text { Région de Bou Ahmed } \\
\text { (Stehat et Chmaala) } \\
\text { (Sur } 41 \text { ans) }\end{array}$} & Zone 1 & 0,0 & 0,0 & Stabilité \\
\hline & Zone 2 & $-50,1$ & $-1,2$ & Erosion \\
\hline & Zone 3 & $+86,5$ & $+2,1$ & Accrétion \\
\hline \multirow{4}{*}{$\begin{array}{l}\text { Région de Jebha } \\
\text { (Sur } 41 \text { ans) }\end{array}$} & Zone 1 & $-30,0$ & $-0,7$ & Erosion \\
\hline & Zone 2 & 0,0 & 0,0 & Stabilité \\
\hline & Zone 3 & $+62,4$ & $+1,5$ & Accrétion \\
\hline & Zone 4 & $-30,0$ & $-7,0$ & Erosion \\
\hline
\end{tabular}




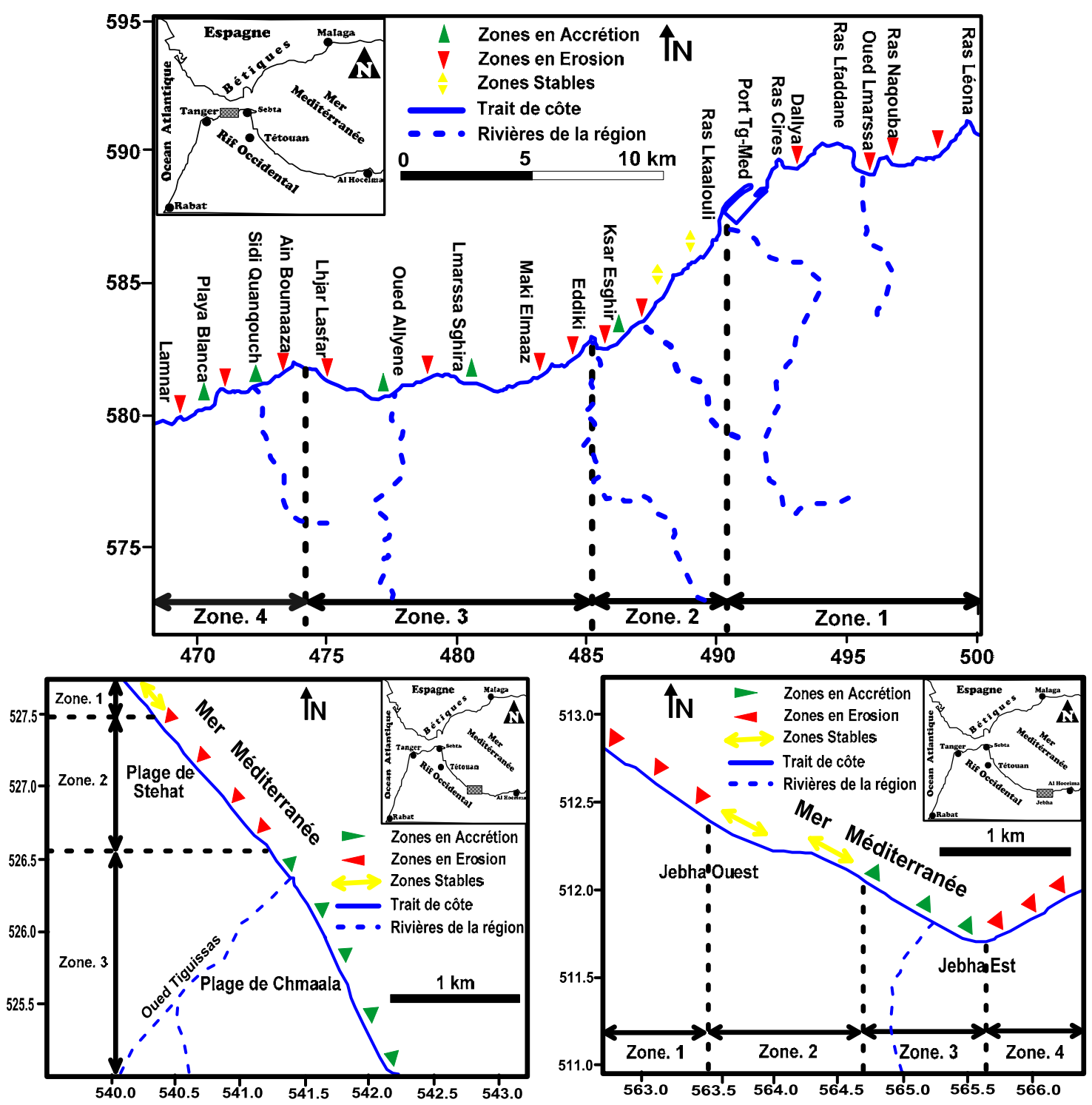

Figure 4. Carte de la variation du trait de côte de la région de Ksar Esghir entre 1966 et 2005, des régions de Chmaala-Stehat et Jebha entre 1969 et 2009.

\subsection{Bilan évolutif des sites de la région de Bou Ahmed entre 1969 et 2009}

La synthèse des résultats souligne les faits suivants (figure 4 et tableau 2) : des zones stables à l'extrême nord de la plage de Stehat (Zone 1), des zones en érosion du reste de la plage de Stehat (Zone 2), avec un taux moyen annuel de l'ordre de -1,2 m/an et des zones en accrétion (plage de Chmaala : Zone 3), avec un taux moyen annuel de l'ordre de $+2,1 \mathrm{~m} / \mathrm{an}$.

\subsection{Bilan évolutif des sites de la région de Jebha entre 1969 et 2009}

La synthèse des résultats souligne les faits suivants (figure 4 et tableau 2) : des zones stables (zones des falaises : Zone 2), des zones en érosion (plage Jebha : Zones 1 et 4), 


\section{XIII ${ }^{\text {èmes }}$ Journées Nationales Génie Côtier - Génie Civil \\ Dunkerque, 2-4 juillet 2014}

avec un taux moyen annuel de l'ordre de $-0,7 \mathrm{~m} / \mathrm{an}$ et des zones en accrétion (plage de Jebha : Zone 3), avec un taux moyen annuel d'environ de $+1,5 \mathrm{~m} / \mathrm{an}$.

\section{Conclusion}

De cette étude il ressort que, les différents sites, selon les zones, de la frange littorale de la région de Ksar Esghir, Bou Ahmed et Jebha, se présente comme un système côtier à équilibre fragile, dominé pour l'essentiel par :

a) Pour les littoraux de la région de Ksar Esghir

- un bilan évolutif global du comportement du trait de côte négatif, sur 40 ans, entre 1966 et 2005, avec environ 63\% des espaces littoraux en érosion contre 37\% des espaces littoraux en accrétion,

- une tendance à l'accrétion très limitée due, pour l'essentiel, aux transferts de matière entre les secteurs, aux apports continentaux excessivement importants durant les années pluviales et aux apports instantanés dus aux phénomènes des glissements de terrain très fréquents dans la région,

- une stabilité limitée à la zone du port Tanger - Med, entre Ras Cires et Ras Lkaalouli.

Cette tendance régressive liée essentiellement et selon l'ordre d'importance des facteurs la régissant à un hydrodynamisme fort, à un impact direct de la dynamique des versants côtiers de la chaîne du Rif et aux effets indirects et directs de l'anthropisation, suite au développement important qu’a connu cette région depuis le début des années 1990.

b) Pour les littoraux de la région méditerranéenne de Bou Ahmed et Jebha

Une tendance évolutive marquée, par l'alternance :

- des zones en érosion, avec un recul total moyen de la ligne du rivage d'environ -50 m sur 41 ans au niveau de la plage de Stehat et -30,0 m/41ans dans les plages de Jebha,

- des zones en accrétion totale moyenne, avec $+86,5 \mathrm{~m}$ sur 41 ans au niveau de la plage de Chmaala, $+62,4 \mathrm{~m}$ sur 41ans et de part et d'autre de l'embouchure de l'oued Ouringa à l'Est de Jebha,

- une stabilité limitée au tronçon sud de la plage ouest de Jebha (Zone 2).

Cette dynamique littorale est le résultat des effets des décharges brutales des cours d'eau de la région (Constats d'accrétion) et des transferts latéraux des matériaux de ces sites côtiers (Constats d'érosion). Ce transfert se fait selon une direction générale Est Ouest à Stehat et à Jebha.

\section{Références bibliographiques}

BENMAKHLOUF M. (1990). Genèse et évolution de l'accident de Tétouan et son rôl transformant au niveau du Rif septentrional (Maroc) (depuis l'Oligocène jusqu'à l'actuel). Thèse 3ème cycle, Faculté des Sciences, Rabat, 162 p.

CHALOUAN A. (1986). Les nappes Ghomarides (Rif septentrional Maroc). Un terrain varisque dans la chaîne Alpine. Thèse Doc. Univ Luis Pasteur, Strasbourg, 317 p. 
CLIMATE-DATA (site web). http://climate-data.org/. Données climatologiques de la région d'El Jebha et Stehat.

DIDON J, DURAND DELGA M., KORNPROBST J. (1973). Homologies géologiques entre les deux rives du détroit de Gibraltar (Espagne). BSGF, 7, T. XV-2, pp 77-105.

DURAND-DELGA M., FONTBOTE J.M. (1980). Le cadre structural de la Méditerranée occidentale. 26th International Geological Congress, Les chaînes alpines issues de la Téthys, Mémoires BRGM, Vol. 11, pp 67- 85.

DURAND-DELGA M., HOTTINGER L., MARCAIS J., MATTAUER M., MILLIARD Y., SUTER G. (1960-62). Données actuelle sur la structure de rif. In Livre-mémoire P. Fallot, Mém. H. Sér. Soc. Géol. Fr., Paris, t. 1, pp 399-422.

EL FADEL L. (2006). Evolution morpho - dynamique et analyse morphostructurale du littoral méditerranéen marocain de Oued Laou (Provinces de Tétouan et Chefchaouen Maroc Nord Occidental). Mémoire de DESA. Université Abdelmalek Essaâdi. 76 p.

EL MEKADEM N., EL MOUTCHOU B., EL FADEL L., EL HAJJAJI K. (2009). Etude morphodynamique et morphostructurale de la région de Bou Ahmed (Province de Chefchaouen - Maroc Nord Occidental). Actes des $5^{\text {ème }}$ J. I. G. E. Fès. Maroc.

EL MEKADEM N., EL MOUTCHOU B., OUAZANI TOUHAMI A., STITOU EL MESSARI J. (2009). Evolution historique, géomorphologique et occupation du sol du littoral méditerranéen de Bou Ahmed (Chefchaouen, Maroc). $5^{\text {ème }}$ JIGE. Fès. Maroc.

EL MOUTCHOU B. (1995). Dynamique côtière actuelle et évolution morpho dynamique de la frange littorale entre M'diq et Oued Laou (province de Tétouan, Maroc Nord Occidental). Thèse de 3ème Cycle, Univ. Mohamed V, Rabat, 165 p.

EL MOUTCHOU B., EL MEKADEM N., EL FADEL L., EL HAJJAJI Kh. (2011a). Evolution historique et géomorphologique de la ligne du rivage de la zone côtières de Bou Ahmed (Chefchaouen - Maroc). Conférence méditerranéenne côtière et maritime. edition 2. Tanger. Maroc, pp 163-168. http://dx.doi.org/10.5150/cmcm.2011.035

EL MOUTCHOU B. EL FADEL L., EL HAJJAJI Kh. (2011b). Evolution morphodynamique et morphosédimentaire du littoral méditerranéen d'Oued Laou (Tétouan - Maroc). Conférence méditerranéenne côtière et maritime. Edition 2. Tanger. Maroc, pp 157-162. http://dx.doi.org/10.5150/cmcm.2011.034

JAAIDI E.B., AHMAMOU M., ZOUGARY R., CHATRE B., EL MOUTCHOU B., MALEK F., NAIM K. (1993). Le littoral méditerranéen entre Tétouan et Ceuta et atlantique entre Tanger et Asilah (Maroc) : Impact des aménagements portuaires sur la dynamique côtière. In Aménagement du littoral et évolution des côtes ; L'environnement côtier marocain en péril. Publ, Com, Nat, Géogr, Maroc, Sept 1993, pp 21-34.

NHALA I. (2013). Les houles dans la péninsule tingitane, de part et d'autre du Détroit de Gibraltar. Mémoire PFE, Univ. Abdelmalek Essaadi, 40 p. 